\title{
EFECTO DE LA GALLINAZA EN PLANTACIONES DE CACAO (THEOBROMA CACAO L.) EN DESARROLLO ${ }^{1}$
}

\author{
María Orozco ${ }^{2}$, Susanne Thienhaus ${ }^{2}$
}

\begin{abstract}
RESUMEN
Efecto de la gallinaza en plantaciones de cacao (Theobroma cacao L.) en desarrollo. Se estudiaron el efecto del abono orgánico gallinaza sobre el crecimiento y desarrollo del cultivo de cacao (Theobroma cacao L.) en estado juvenil.El ensayo inició en febrero de 1993, en tres fincas con plantaciones comerciales de 20 meses de edad, ubicadas en el municipio El Rama, Región V Nicaragua. Durante catorce meses se realizaron cuatro aplicaciones de fertilizantes, comparando tres niveles de gallinaza (454 g, 908 g y 1,362 g/árbol/ aplicación) un testigo de fertilización mineral (tres aplicaciones de 100 g/árbol de la fórmula comercial 15-15-15, más una aplicación de $100 \mathrm{~g} /$ árbol de urea al $46 \%$ ) y un testigo absoluto sin aplicación. Se utilizó un diseño de bloques completos al azar evaluando las variables con análisis de varianza, separación de medias según Duncan y análisis de regresión y correlación. Los resultados demostraron que la aplicación de $1.362 \mathrm{~g}$ de gallinaza obtuvo un efecto estadísticamente superior sobre la producción inicial del cacao y sobre el incremento del diámetro de tallo. La aplicación de 454 g y 908 g de gallinaza resultó estadísticamente igual a la fertilización mineral. En cuanto a altura de planta no se detectaron diferencias estadísticas entre los tratamientos. El tratamiento de fertilización presentó el menor efecto sobre el crecimiento y producción inicial del cultivo de cacao.
\end{abstract}

\begin{abstract}
The effect of three rates of chicken manure on the growth and development of cocoa (Theobroma cacao L.) trees. We studied the effect of the organic chicken manure on the growth and development of cocoa trees (Theobroma cacao L.), during their juvenile stage. The experiment was initiated in February, 1993, in three commercial plantations, 20 months old, located in the county of El Rama, Region V. During the following 14 months we carried out three application ,comparing, the three levels of chicken manure $(454,908$. and 1,362 g per tree and application) with mineral fertilizer (three applications , of 15-15-15fertilizers $100 \mathrm{~g}$ per tree, plus one application of, urea $100 \mathrm{~g}$ per tree), and a check without any applications. A random complete bolck design was utilized and means were compared by, Duncan's test and regression and correlation analyses were also made. The results showed that with the application of $1,362 \mathrm{~g}$ of chicken manure, a significatively higher effect over the initial production of cocoa, and an increase of the stalk diameter were observed. The applications of 454 and 908, of chicken manure resulted in production levels equal to those of the treatment with mineral fertilizers. In relation to plant height, we did not find statistical differences among treatments. The treatment without fertilizer, showed the smallest growth and lower production.
\end{abstract}

\section{INTRODUCCION}

El cacao (Theobroma cacao L.) es originario de América, los indios lo cultivaban en México y Centro América (Blanco, 1984). Actualmente es producido en 21 países, los que alcanzan una producción anual de 2,3 millones de toneladas de almendras de cacao (ICCO, 1993). Según datos de los zonales del MAG y de INRA, la distribución actual de las áreas de cacao en Nicaragua se concentra en 6.195 ha en cinco departamentos. La producción actual total se estima en aproximadamente 1,000 toneladas anuales. El volumen promedio exportado por año, es de $522 \mathrm{t}$ (Thienhaus, 1992).

Los requerimientos nutricionales del cacao varían de acuerdo con el material de siembra y las condiciones del cultivo, especialmente el grado de sombreamiento. La máxima producción de cacao resultará de la combinación de un sombrío moderado con un suelo de fertilidad natural alto o adecuadamente fertilizado (Compañía Nacional de Chocolates, 1988).

1 Presentado en la XLI Reunión Anual del PCCMCA en El Salvador, Centroamérica. 27 al 31 de marzo, 1996.

2 Universidad Nacional Agraria, Facultad de Agronomía, Escuela de Producción Vegetal. Managua, Nicaragua. 
Una encuesta realizada en 1992 en todo el país reveló, sin embargo, que el 65 por ciento de los productores entrevistados nunca han fertilizado sus plantaciones de cacao, tanto por razones económicas como por desconocimiento general sobre el uso de fertilizantes. También, el estudio reveló que los productores que aplican fertilizantes dos a cuatro veces al año logran obtener una producción de 0,89 t/ha contra un promedio general de 0,47 t/ha (Thienhaus, 1992).

Nicaragua es un país dependiente de los fertilizantes minerales, por lo que se orienta realizar evaluaciones de fertilizantes orgánicos de origen animal, como la gallinaza que en nuestro país se desperdicia, o más aún, contribuye a la contaminación del medio ambiente, especialmente del recurso acuífero.

El excremento de gallinas (Gallus domésticus) de granja contiene más nutrimentos para la planta que cualquier otro estiércol, sin embargo requiere un manejo eficaz para que no pierda rápidamente el nitrógeno. Varias razones explican la insignificante utilización de gallinaza, entre otras, la falta de información acerca de su valor como abono y los cuidados que necesita, el desconocimiento de cómo y cuándo utilizarlo (Agricultura de las Américas, 1965). Actualmente, algunos granjeros están considerando la posibilidad de comercializar la gallinaza ya que la demanda de este estiércol está incrementándose.

Con este trabajo se pretende resolver parcialmente el problema de fertilización en las plantaciones cacaoteras de los pequeños productores, especialmente aquellos beneficiarios del Proyecto Promundo Humano del municipio El Rama, considerando la nutrición del cultivo como un factor integral del sistema de manejo de finca. Esto permitiría al productor prescindir de recursos externos, aprovechando entre subproductos de otros rubros de producción de la finca y crear de esa manera, un sistema sostenible de explotación a largo plazo.

El presente trabajo se realizó con los siguientes objetivos:

1- Encontrar el efecto de las diferentes dosis de gallinaza, con respecto al crecimiento del cultivo de cacao y las primeras floraciones y fructificaciones. 2Determinar las cantidades adecuadas de aplicación de gallinaza, como abono orgánico y establecer comparaciones económicas con el fertilizante mineral y el testigo absoluto.3- Determinar a través de las observaciones comparativas y los análisis de suelo, los posibles cambios en las propiedades químicas y físicas del suelo.

\section{MATERIALES Y METODOS}

\section{Antecedentes del área experimental}

Entre 1990 y 1991 el Proyecto Promundo Humano realizó la siembra de 42 ha de cacao con el objetivo de apoyar a los pequeños productores de la zona de El Rama en el establecimiento de un sistema agroforestal distribuido en un área de 0,7 ha por productor brindándoles semillas e insumos, incluyendo fertilizantes, y asistencia técnica para el manejo del cultivo durante los dos primeros años.

Inicialmente esas tierras eran desmontadas (tacotales) y fueron utilizadas para cultivos anuales con barbechos intermedios. En 1990 se sembraron musáceas (Musa spp) a una distancia de $3 \times 3 \times 6 \mathrm{~m}$ como sombra temporal, en el mismo año se estableció la sombra permanente utilizando pejibaye (Bactris gassipaes H.B.K.) a una distancia de 6 x $12 \mathrm{~m}$ y especies leguminosas como madero negro Gliricidia sepium (Jacq) Steud, guabo (Inga sp.) y poró Erythrina fusca (Walpes) O.F. Cook, a una distancia de 6 x 12 m de manera intercalada. En julio - agosto de 1991 se trasplantó el cacao a una distancia de $3 \times 3 \mathrm{~m}$, utilizando semillas híbridas provenientes del Centro Experimental El Recreo.

\section{Descripción del lugar y experimento}

El experimento se realizó en el municipio El Rama, Región V, en tres fincas, ubicadas en las orillas del Río Mico. Su ubicación geográfica está entre los $12^{\circ} 7^{\prime}$ latitud norte y $84^{\circ} 24^{\prime}$ longitud oeste. La duración del ensayo en el campo abarcó el período comprendido entre febrero de 1993 - abril de 1994

El ecosistema según Holdridge (1967), se clasifica como bosque tropical húmedo (BTH), cuyas condiciones se presentan en el Cuadro 1.

Los suelos en que se estableció el experimento son de tipo aluvial, caracterizándose por una textura franco-

Cuadro 1. Condiciones climáticas del área del municipio El Rama.

\begin{tabular}{ll}
\hline Precipitación media anual & $3,026 \mathrm{~mm} / \mathrm{año}$ \\
Temperatura media anual & $25,4{ }^{\circ} \mathrm{C}$ \\
Humedad relativa anual & $78,3 \%$ \\
Altitud & $15-25 \mathrm{msnm}$ \\
Radiación solar media diaria & 4 horas 13 minutos \\
\hline * Fuente: Estación Meteorológica del Centro Experimen- \\
$\quad$ tal El Recreo (1954 - 1992).
\end{tabular}


arcillosa, un $\mathrm{pH}$ entre 4.5 y 6.5 , alto en contenido de materia orgánica y bajo en fósforo.

La clasificación de los suelos de El Recreo corresponden a los sub-grupos taxonómicos: Molisoles; Tipic haplustolls; Alfisoles; Udic haplustalfs + Ultic haplustalfs y Ultic tropudalfs (Malespín, 1983).

\section{Tratamientos}

Los tratamientos estudiados consistieron en tres niveles de aplicación de gallinaza seca de 10 meses de almacenamiento, procedente de la Granja Avícola La Estrella, Managua, Km 22 1/2 carretera Norte, más un testigo de fertilización mineral y un testigo absoluto ( $\sin$ aplicación), según se describe a continuación.

$\mathrm{T} 1=454 \mathrm{~g}$ de gallinaza por planta por aplicación.

$\mathrm{T} 2=908 \mathrm{~g}$ de gallinaza por planta por aplicación.

$\mathrm{T} 3=1,362 \mathrm{~g}$ de gallinaza por planta por aplicación.

$\mathrm{T} 4=100 \mathrm{~g}$ de fertilizante químico (15-15-15) en tres aplicaciones más 100 g de urea en la última fertilización.

T5 = Testigo absoluto. Los tratamientos con gallinaza se aplicaron 4 veces en un lapso de 14 meses.

En febrero de 1993, antes de iniciar el ensayo se realizó en el Laboratorio de suelos y aguas de la Universidad Nacional Agraria (UNA), un análisis de gallinaza provenientes de tres fuentes, estiércol con cascarilla de arroz, gallinaza de ponedoras y gallinaza de pollos de engorde (procesada). En el ensayo se utilizó gallinaza de ponedoras por su mayor contenido de calcio y disponibilidad del material, se establecieron tres niveles de aplicación de gallinaza: $454 \mathrm{~g}, 908 \mathrm{~g}$ y 1,362 g equivalentes a 1,2 y 3 libras. Los resultados se muestran en el Cuadro $2\left(\mathrm{P}_{2} \mathrm{O}_{5}\right)$.

El contenido de ácido fosfórico en la gallinaza según Yágodin (1988), el porcentaje de masa húmeda muestra un valor alto de 1,8\%. De igual manera el estudio realizado por Voitl, Guggenberg, Willi, 1980, indica un rico contenido de ácido fosfórico de $1.5 \%$. El análisis realizado en la Universidad Nacional Agraria de gallinaza seca mostró un porcentaje de $0.39 \%$ de fósforo, es decir, un $0.89 \%$ de ácido fosfórico. No se determinó el porcentaje de materia orgánica que presentaba esta muestra. Según Agricultura de las Américas (1965), el porcentaje de materia orgánica para gallinaza fresca es $19,10 \%$ y 20,86 $\%$ para gallinaza de 10 semanas. Gallinaza fresca exenta de humedad constató un 79,59\% de materia orgánica y un $64,15 \%$ para gallinaza de 10 semanas.

Dado que la dosis recomendada para cacao en suelos con bajo contenido de fósforo es de $60 \mathrm{~kg} / \mathrm{ha}$ y tomando en cuenta que la gallinaza posee un $0,39 \%$ de fósforo, se consideró necesario aplicar la cantidad de 15 $\mathrm{t}$ de gallinaza por año. Sin embargo, esta cantidad se encuentra fuera del rango económico posible, por lo que se decidió determinar las dosis de aplicación según costos de adquisición y transporte del material orgánico y que éste se encuentre con margen rentable en comparación con la aplicación del fertilizante químico.

Las plantas al momento de la primera aplicación tenían una edad de 20 meses y al terminar la última aplicación, 34 meses.

La aplicación se realizó en forma localizada en el área de goteo (caseo) de las plantas de cacao, en el área comprendida entre los radios de 30 y $50 \mathrm{~cm}$ a partir de la base del tallo, posteriormente se cubrió el abono con la maleza seca.

La aplicación anual se realizó en tres momentos, iniciándose en el mes de febrero aprovechando las últimas lluvias del invierno, con el objetivo de proporcionar vigor a las plantas durante la estación seca. La segunda aplicación se realizó en junio con el objetivo de fortalecer a la planta durante el proceso de brotación y formación de botones florales. La aplicación de septiembre coincidió con el inicio de floración y fructificación en las diferentes parcelas. En el mes de febrero del 94 se realizó la primera aplicación del siguiente ciclo. El ensayo finalizó con la toma de datos realizada en los últimos días del mes de abril de 1994.

Cuadro 2. Características químicas del abono orgánico gallinaza.

\begin{tabular}{lccrcrrrrr}
\hline \multicolumn{1}{c}{ Identificación } & \multicolumn{1}{c}{ Porcentaje } \\
\cline { 2 - 10 } & $\mathbf{P}$ & $\mathbf{K}$ & $\mathbf{K a}$ & $\mathbf{M g}$ & $\mathbf{F e}$ & $\mathbf{M n}$ & $\mathbf{K u}$ & $\mathbf{Z n}$ \\
\hline Cascarilla de arroz (pollitos) & 0,001 & 0,25 & 0,28 & 0,11 & 0,042 & 0,018 & 0,000 & 0,001 \\
Gallinaza de ponedoras * & 0,390 & 0,59 & 60,10 & 0,82 & 0,084 & 0,034 & 0,001 & 0,021 \\
Gallinaza de engorde (procesada) & 0,350 & 0,64 & 56,40 & 0,92 & 0,061 & 0,038 & 0,002 & 0,0026 \\
\hline
\end{tabular}

* Gallinaza utilizada en el experimento

Fuente: Laboratorio de Suelos y Aguas de la Universidad Nacional Agraria UNA (1993). 


\section{Diseño experimental}

El diseño utilizado, fue bloques completos al azar (BCA) con cinco tratamientos, con tres repeticiones en dos productores y una réplica en un tercer productor, para un total de siete réplicas.

Al realizar los análisis estadísticos, se determinó el análisis de varianza (ANDEVA), la separación de medias según Duncan y el análisis de regresión y correlación entre las variables diámetro de tallo, respecto al porcentaje de fructificación y número de frutos prendidos por tratamiento y niveles de aplicación respecto al incremento en diámetro de tallo, porcentaje de fructificación y número de frutos prendidos por tratamiento.

\section{Descripción de la parcela experimental}

\section{Productor 1. Emilio Fajardo}

Distancia de siembra: $3 \times 3 \mathrm{~m}$

Parcela experimental: 36 plantas en cuadro $(6 \times 6$ plantas $)=324 \mathrm{~m}^{2}$

Parcela útil: $\quad 16$ plantas en cuadro

Área total: $\quad 3744 \mathrm{~m}^{2}$

Productor 2. Valentín Rocha

Distancia de siembra: 3 × $3 \mathrm{~m}$

Parcela experimental: 35 plantas en rectángulo $(5 \times 7$ plantas $)=315 \mathrm{~m}^{2}$

Parcela útil: $\quad 15$ plantas en rectángulo

$(3 \times 5$ plantas $)=135 \mathrm{~m}^{2}$

Área total: $\quad 3591 \mathrm{~m}^{2}$

Productor 3. Margarito Martínez

Distancia de siembra: $3 \times 3 \mathrm{~m}$

Parcela experimental: 36 plantas en cuadro

(6 6 6 plantas $)=324 \mathrm{~m}^{2}$

Parcela útil: $\quad 16$ plantas en cuadro

Área total: $\quad 1404 \mathrm{~m}^{2}$

Se formaron bordes comunes entre parcelas y bordes comunes entre bloques, reduciéndose de esta forma el área total por productor.

\section{Variables evaluadas en el experimento}

- Diámetro del tallo ( $\mathrm{mm})$, se tomó cada tres meses a una altura de $8 \mathrm{~cm}$ de la superficie del suelo en todas las plantas de la parcela útil, se calculó el incremento del diámetro (feb. 93- Abr. 94) y luego análisis de regresión del diámetro en función de los niveles de aplicación de gallinaza.

- Altura de planta $(\mathrm{cm})$, se tomó cada tres meses midiendo desde la superficie del suelo hasta el ápice del brote más alto en todas las plantas de la parcela útil y luego se calculó el incremento de la altura durante el período experimental.

- Floración (\%), se registró la cantidad de plantas con flores por parcela útil cada tres meses y luego se calculó el porcentaje de árboles en floración.

- $\quad$ Fructificación (\%), se registró el número de frutos prendidos por árbol por parcela útil cada tres meses, para evaluar:

El porcentaje de árboles en fructificación

El número de frutos prendidos por parcela.

Para la interpretación de estos resultados, se hizo una transformación a los porcentajes de floración, fructificación y número de frutos prendidos por parcela utilizando la fórmula

$\mathrm{Y}=$ raíz cuadrada $(\mathrm{x}+.5)$, para reducir el coeficiente de variación y detectar diferencias estadísticas.

Se realizó análisis de regresión y correlación entre las variables que presentaron significancia estadística:

- Diámetro de tallo con porcentaje de fructificación y número de frutos.

- Niveles de aplicación con porcentaje de fructificación y número de frutos.

- Contenido de macroelementos del suelo. En las parcelas donde se aplicaron los tratamientos de fertilización orgánica (tercer nivel), fertilización química y testigo absoluto se realizó un análisis de suelo para auxiliarse en la interpretación de los resultados y se aplicó un análisis de regresión para las variables de suelo que presentaron correlación significativa entre ellas.

Se tomaron tres submuestras por tratamiento en cada una de las siete repeticiones y se formó una muestra compuesta por tratamiento y por productor para establecer comparaciones simple entre los resultados. La muestra se tomó en el área radicular de las plantas de cacao.

A nivel de observación se anotó la característica de color del suelo de las diferentes capas a $30 \mathrm{~cm}$ de profundidad.

\section{Manejo experimental}

El control de malezas se realizó manualmente en forma de chapias y caseos cada tres meses, una semana antes de la aplicación del fertilizante.

La regulación de sombra se realizó al inicio del período lluvioso en forma de deshije y deshoja de las 
musáceas (sombra temporal) y en forma de poda de formación de los árboles de sombra permanente, madero negro, guabo y poró (Gliricidia sepium, Inga sp. y Erythrina fusca respectivamente).

La poda de formación se realizó al inicio de la estación lluviosa y se repitió en el mes de octubre.

El deschuponado se realizó cada dos meses. No se realizó aplicación química contra plagas y enfermedades, ya que no se presentaron problemas fitosanitarios económicamente importantes, salvo algunos casos de ataque de insectos minadores (Xyleborus sp.) en combinación con mal de machete (Ceratocystis fimbriata Ell. \& Halst) a los cuales se aplicó poda sanitaria.

Se presentaron problemas de pérdidas de plantas, debido a fenómenos naturales, como fueron las tormentas Bert y Gert que provocaron inundaciones en las plantaciones de cacao.

\section{RESULTADOS Y DISCUSION}

\section{Efecto de las diferentes dosis de gallinaza sobre el crecimiento y desarrollo del cultivo}

\section{Diámetro de tallo}

En el Cuadro 3, se presentan los efectos de los tratamientos respecto a los incrementos en grosor del tallo. El análisis de varianza realizado a los incrementos del período febrero 1993 - abril 1994, mostró diferencias significativas (5\% de probabilidad) presentando el tratamiento tres (1,362 g de gallinaza) los mayores valores, siendo superior a los demás tratamientos a partir del mes de setiembre; es decir, seis meses después de la primera aplicación.

Es importante señalar que para el mes de junio la gallinaza no se había incorporado completamente al suelo por su característica como abono orgánico de descomposición lenta, acentuado por la falta de humedad durante el verano.

El testigo absoluto, a partir de la toma de datos del mes de setiembre, reveló el menor incremento respecto a los demás tratamientos. Estos resultados permiten señalar la necesidad de fertilización en el cultivo de cacao y que la gallinaza favorece la nutrición del árbol proporcionándole mayor vigor a la planta.

En relación al análisis de regresión entre los factores diámetro de tallo (incrementos febrero 1993-abril 1994) y niveles de aplicación, se obtuvo una ecuación $\mathrm{y}=21,705+1,891 \mathrm{x}$, el coeficiente de correlación $\mathrm{r}=$ $0,405, \mathrm{r} 2=0,164$ indica que un $16,4 \%$ de la variación del diámetro depende de los tratamientos aplicados.

\section{Altura de planta}

La altura de la planta de cacao es una variable de comportamiento muy irregular, ya que está muy influenciada por el genotipo de la planta y el manejo durante la poda.

Realizado el análisis de varianza a los promedios de altura de planta, se encontró que no existen diferencias significativas entre los tratamientos en estudio.

Puede notarse en el comportamiento de esta variable, que a partir del mes de setiembre los tratamientos muestran algún efecto, presentando el mayor promedio 195,31 cm el tratamiento con 1,362 g de gallinaza. En enero de 1994 los mayores promedios los obtuvieron los tratamientos de 1,362 g de gallinaza con 213,36 cm y fertilización química con 207,04 cm de altura. En abril, prácticamente se mantuvo el tamaño de las plantas estudiadas presentando los mayores promedios de $217,45 \mathrm{~cm}$ y $217,89 \mathrm{~cm}$, los tratamientos con $908 \mathrm{~g}$ y 1,362 g de gallinaza de forma respectiva.

Este comportamiento se debe a que durante la época seca las plantas presentan menor crecimiento

Cuadro 3. Incremento en el diámetro del tallo (en mm) en el cultivo del cacao (febrero 1993 - abril 1994).

\begin{tabular}{cccccc}
\hline Tratamientos & \multicolumn{5}{c}{ Meses } \\
\cline { 2 - 6 } & feb-jun & jun-sep & sep-ene & ene-abr & feb 93-abr 94 \\
\hline T1 & 3,95 & 6,51 & 6,69 & 5,05 & $23,72 \mathrm{ab}$ \\
T2 & 5,75 & 7,30 & 7,09 & 5,60 & $25,17 \mathrm{ab}$ \\
T3 & 4,73 & 7,79 & 8,45 & 6,57 & $27,54 \mathrm{a}$ \\
T4 & 3,78 & 5,95 & 7,56 & 6,02 & $24,47 \mathrm{ab}$ \\
T5 & 4,61 & 5,68 & 6,54 & 4,63 & $21,73 \quad \mathrm{~b}$ \\
\hline
\end{tabular}


vegetativo, debido a que hay menor humedad en el suelo, disminuye la taza de descomposición de la materia orgánica, la absorción de nutrientes por la planta y la formación de tejidos vegetativos.

En el Cuadro 4, se presenta la respuesta de los tratamientos sobre el incremento de la altura de planta a 14 meses de iniciado el ensayo.

Es necesario considerar que esta variable se vio afectada por factores antropogénicos (daños mecánicos realizados por el hombre), vientos, inundaciones. También se realizaron podas sanitarias como método de control de Xyleborus sp y el mal del machete.

La altura de planta es una variable menos confiable que el diámetro de tallo, debido a que éste último está menos afectado por daños mecánicos. EL coeficiente de variación fue mayor para la altura de planta 30,49\% que para el diámetro de tallo $17.54 \%$ durante todo el período de evaluación del ensayo, lo que demuestra que la variable diámetro de tallo resulta más confiable por las razones antes mencionadas. Sin embargo, se observa una influencia de los fertilizantes sobre la altura de planta, ya que los análisis numéricamente muestran que el menor crecimiento de altura de planta durante el período feb./93 y abr./94, lo presentó el testigo absoluto, que no recibió ninguna aplicación.

\section{Porcentaje de floración}

Los resultados obtenidos del análisis de varianza, no mostraron diferencias significativas entre los tratamientos.

En el mes de setiembre (26 meses después del trasplante) se encontraron las primeras plantas en floración. A esta fecha se habían realizado dos aplicaciones de los diferentes tratamientos en estudio.

Los resultados del mes de setiembre mostraron numéricamente que el tratamiento de $908 \mathrm{~g}$ y 1,362 g de gallinaza respectivamente, presentaron los mayores porcentajes de plantas en floración en relación a los demás tratamientos .
Los datos tomados el mes de enero revelaron que el tratamiento de 1,362 g de gallinaza superó al tratamiento de $908 \mathrm{~g}$ en $19.87 \%$ y al testigo absoluto en un 46,21\% (Figura 4). En el mes de abril el tratamiento con fertilizante químico fue superado por el tratamiento de $454 \mathrm{~g}$ de gallinaza. El tratamiento de 1,362 g de gallinaza se mantuvo con el mayor número de plantas en floración superando al testigo absoluto en un 42,23\%.

El tratamiento de 908 g no logró aumentar el porcentaje de plantas floreciendo. Este comportamiento posiblemente se debe a la influencia de varios factores como: precocidad de los genotipos, efecto de sombra, factores naturales.

El tratamiento cinco (testigo absoluto) durante el período de setiembre a abril, mostró el menor porcen-taje de plantas con flores. Lo que puede atribuirse a que las parcelas no recibieron ningún tipo de fertilización adicional, demostrando de esa manera la importancia de la fertilización del cultivo.

Comparando estos resultados con los obtenidos por Thienhaus (1986), quién estableció un ensayo en el Centro Experimental El Recreo con los tratamientos: Estiércol descompuesto, AIC (Abono integral completo, distribuido por Fertiquasa, Esquipulas elaborado a base de gallinaza), fertilizante completo, estiércol más fertilizante completo, se encontró que a los 20 meses después del trasplante algunos tratamientos mostraron el inicio de la floración y que existió una correlación entre el diámetro y el porcentaje de árboles en floración entre los 20-24 meses luego del trasplante.

Se observó que los tratamientos con AIC (primer nivel) y estiércol con fertilizante químico (segundo nivel) mostraron influencias de la fuente de fertilizante sobre la precocidad de floración y fructificación. Se puede suponer que la mayor precocidad presentada por las plantas del ensayo de Thienhaus (1986) se debió a que los tratamientos se aplicaron desde la época de vivero y en la plantación establecida.

Cuadro 4. Incremento en la altura de planta (cm) en el cultivo del cacao (febrero 1993 - abril 1994).

\begin{tabular}{cccccc}
\hline \multirow{2}{*}{ Tratamientos } & \multicolumn{2}{c}{ Meses } & & & \\
\cline { 2 - 6 } & Feb-Jun & Jun-Sep & Sep-Ene & Ene-Abr & Feb 93-Abr 94 \\
\hline T1 & 14,73 & 29,35 & 20,03 & 9,36 & $73,49 \mathrm{a}$ \\
T2 & 28,54 & 23,51 & 20,95 & 15,19 & $88,19 \mathrm{a}$ \\
T3 & 15,72 & 36,68 & 18,05 & 3,53 & $74,17 \mathrm{a}$ \\
T4 & 15,57 & 30,25 & 32,18 & 0,03 & $78,04 \mathrm{a}$ \\
T5 & 15,52 & 22,52 & 19,42 & 11,85 & $69,31 \mathrm{a}$ \\
\hline
\end{tabular}


En el presente ensayo se presentó cierta precocidad en el inicio de la floración de las plantas (26 meses después del trasplante). Puede observarse en el comportamiento de los tratamientos con gallinaza y fertilizante químico, un efecto similar sobre el inicio de la floración, superando al tratamiento testigo absoluto. Sin embargo, esta variable fue afectada por factores adversos como son las inundaciones en los meses de agosto y septiembre de 1993, que provocaron un comportamiento irregular de los tratamientos sobre la floración de las plantas de cacao.

\section{Porcentaje de fructificación.}

En el presente trabajo las primeras plantas en fructificación se presentaron en el mes de enero ( 29 meses después del trasplante). La evaluación de porcentaje de plantas en fructificación en cada uno de los tratamientos en estudio mostró diferencias significativas en el mes de abril (Cuadro 5).

Hay que considerar que el porcentaje de plantas con frutos encontradas en enero fue reducida anteriormente por fenómenos naturales como fueron las inundaciones causadas por las tormentas Bert (agosto 1993) y Gert (setiembre 1993) que afectó a toda la zona.

Los datos del mes de enero indican que las parcelas donde se aplicó el tratamiento de $454 \mathrm{~g}$ de gallinaza superó a los demás tratamientos, y al testigo absoluto en un $73,28 \%$.

En el mes de abril se observó que el tratamiento con $1,362 \mathrm{~g}$ de gallinaza tuvo un efecto significativo, superando al tratamiento con $454 \mathrm{~g}$ en $35,42 \% \mathrm{y}$ al testigo absoluto en $134 \%$. Esto permite suponer que existe una mayor asimilación de nutrientes del suelo por las plantas, por el mayor contenido de materia orgánica aplicada, proporcionando al suelo una mejor estructura y mayor actividad de la fauna edáfica.

Cuadro 5. Efecto de los tratamientos sobre el porcentaje de plantas en fructificación (datos transformados).

\begin{tabular}{lcl}
\hline Tratamientos & \multicolumn{2}{c}{ MESES } \\
\cline { 2 - 3 } & Enero 94 & Abril 94 \\
\hline T1 & $2,53 \mathrm{a}$ & $2,23 \mathrm{ab}$ \\
$\mathrm{T} 2$ & $1,78 \mathrm{a}$ & $2,09 \mathrm{ab}$ \\
$\mathrm{T} 3$ & $2,09 \mathrm{a}$ & $3,02 \mathrm{a}$ \\
$\mathrm{T} 4$ & $2,02 \mathrm{a}$ & $1,76 \mathrm{ab}$ \\
$\mathrm{T} 5$ & $1,46 \mathrm{a}$ & $1,29 \mathrm{~b}$ \\
C.V $(\%)$ & 67,38 & 62,31 \\
\hline
\end{tabular}

\section{Número de frutos prendidos}

Respecto al número de frutos no se encontró diferencias significativas en el mes de enero. Sin embargo, el tratamiento con 1,362 g de gallinaza obtuvo el mayor número de frutos seguido del tratamiento $454 \mathrm{~g}$ y el que presentó menor número de frutos fue el tratamiento $908 \mathrm{~g}$, éste fue superado en $78.64 \%$ por el tercer nivel de gallinaza $(1,362 \mathrm{~g})$. En el mes de abril se muestran diferencias estadísticamente significativas resultando el tratamiento con 1,362 g superior al tratamiento cinco (testigo absoluto) en un 150,5\%, mientras que los tratamientos $454 \mathrm{~g}$ y $908 \mathrm{~g}$ de gallinaza fueron estadísticamente igual al fertilizante químico y superior al testigo absoluto .

Estos datos demuestran que existe influencia de la fuente de fertilizante orgánico (gallinaza), sobre la precocidad de floración y fructificación, por ende sobre el número de frutos prendidos que presentaron las parcelas tratadas con este material. Lo que podría explicarse con que las raíces se encuentran en condiciones de mayor capacidad de absorción de nutrientes y agua por factores como: Aireación, mayor retención de humedad, mayor capacidad de intercambio catiónico (CIC) y mayor actividad microbiana (Arzola, Fundora, Machado, 1986).

El menor efecto del fertilizante químico permite suponer que existen pérdidas por lixiviación, volatización y fijación del fósforo inorgánico. Es notoria la importancia de la fertilización del cultivo, para obtener una mayor producción. Esto lo demuestra el testigo absoluto, que no recibió ninguna aplicación y que presentó la menor fructificación.

Respecto al análisis de regresión realizado entre el porcentaje de fructificación y niveles de aplicación de gallinaza, se obtuvo una ecuación lineal de regresión y= $1.806+2.908 x$, el coeficiente de correlación $\mathrm{r}=0,439$, y un coeficiente de determinación r2 $=0,193$, indica que el 19,3\% de la variación en el porcentaje de fructificación está determinado por los niveles de aplicación de gallinaza. Se encontró una ecuación lineal de regresión y= $0,214+2,357 x$, entre los factores número de frutos y niveles de aplicación, $\mathrm{r}=0,389, \mathrm{r} 2=0,152$ que indica que un $15,2 \%$ de la variación depende de los niveles de abono orgánico aplicados .

Análisis de regresión y correlación entre diámetro de tallo y producción inicial del cacao.

El análisis de regresión realizado con los factores diámetro de tallo y porcentaje de fructificación en cacao, resultó altamente significativo, se obtuvo una ecuación lineal $\mathrm{y}=0,119 \mathrm{x}$, un coeficiente de correlación $\mathrm{r}=0.693$. 
El coeficiente de determinación $r 2=0,480$, indica que la variación en el porcentaje de plantas en fructificación depende en un $48 \%$ del diámetro de tallo .

También muestra la correlación significativa que existe entre el número de frutos prendidos y el diámetro, se encontró una ecuación lineal de regresión y $=0,070 \mathrm{x}$, $\mathrm{r}=0,528$ y el coeficiente de determinación $\mathrm{r} 2=0,279$, indica que el $27,9 \%$ de la variación en el número de frutos está determinado por la variable de diámetro de tallo. Para el análisis de estas variables se utilizaron valores sin transformación.

\section{Evaluación económica de los tratamientos.}

El análisis económico resultó difícil, debido a que las plantaciones están apenas entrando en producción y cualquier tipo de fertilización debe considerarse parte de la inversión inicial la cual puede evaluarse económicamente durante todo el período de recuperación de la inversión o sea durante el tercer al quinto año del cultivo. Por lo tanto, el presente análisis se limita a comparar los costos de aplicación de gallinaza con los costos de aplicación del fertilizante químico y los efectos comparativos de ambos sobre la producción inicial del cacao.

Se encontró que los costos de fertilización mineral corresponde a la aplicación de 3,686.8 kg (81,11 qq)/ ha/año de gallinaza equivalente a una dosis de $0,82 \mathrm{~kg}$ (1,8 lbs) de gallinaza/planta/ aplicación (Cuadro 6).

El análisis de regresión con la variable dosis de fertilizantes y porcentaje de plantas en fructificación, número de frutos prendidos e incremento del diámetro de tallo, indica que los costos de la fertilización mineral son mayores que los costos de la fertilización orgánica (gallinaza), en un $118 \%, 187 \%$ y $24 \%$ respectivamente, para las variables antes mencionadas (Cuadro 7).

Según Agricultura de las Américas (1965) en la Estación Experimental Agrícola de Nueva Jersey se realizó un estudio acerca de la forma final en que quedan utilizados los alimentos que consumen las gallinas ponedoras. Si se inicia desde el día en que el pollito sale del cascarón una gallina Leghorn blanca de $1,8 \mathrm{~kg}$ cuando llega a los dos años de edad habrá consumido $62 \mathrm{~kg}$ de alimento. Durante ese período habrá producido 240 huevos, con promedio de $60 \mathrm{~g}$, cada uno y habrá evacuado $118 \mathrm{~kg}$ de gallinaza.

El estudio revela que solamente, $19 \%$ de nitrógeno (N), $12 \%$ de fósforo (P2O5), y $5 \%$ de potasio (K2O) contenidos en el alimento formaron parte en la formación del cuerpo de la gallina y de los huevos. El resto - 81
$\%$ de nitrógeno, $88 \%$ de fósforo y $95 \%$ de potasio fueron evacuados en excremento o gallinaza. El trabajo desarrollado en Nueva Jersey también indica que una gallina excreta aproximadamente $1 \mathrm{~kg}$ de gallinaza por cada $500 \mathrm{~g}$ de alimento seco que consume (Agricultura de las Américas, 1965).

Estos resultados permiten concluir que el productor necesita de un gallinero de aproximadamente 46 gallinas para producir la cantidad de gallinaza/año/ha que equivale económicamente a la fertilización mineral siendo ésta de $0,828 \mathrm{~kg} /$ planta/aplicación. Además el productor aprovechará la producción de huevos y carne de las gallinas. Pero debe considerarse la importancia de una infraestructura adecuada que permita la recolección de los excrementos a nivel de finca y una alimentación adecuada.

Actualmente en el Centro Experimental El Recreo, se está realizando un estudio sobre la producción de concentrado para aves a base de harina de pejibaye como posible alternativa para la crianza de aves de corral en las fincas que poseen el modelo agroforestal estudiado.

\section{Efecto de los tratamientos sobre las propiedades químicas y físicas del suelo}

La mayoría de los suelos del área de la cuenca del Río Escondido pueden ser considerados como ácidos. Esta característica está asociada con procesos que disminuyen la adsorción de ciertos elementos, favorecen la toxicidad del hierro y del aluminio, así como también deficiencia de calcio, magnesio y potasio.

Las bajas cantidades de fósforo disponible encontrada en la mayoría de los suelos pueden atribuirse a la naturaleza ácida del extractor y probablemente a la predominancia de fósforo, bajo las formas de fosfato de hierro y aluminio (Tahal Consulting, 1978).

Fassbender (1984), citado por Thienhaus (1986), considera que el fósforo es el elemento de mayor importancia para el proceso fenológico. Posiblemente la materia orgánica aplicada evitó la fijación del fósforo en la solución del suelo. El encalado constituye indiscutiblemente la práctica más utilizada en los suelos pobres y muy ácidos del trópico húmedo, mediante la cual se restablece en cierta medida el equilibrio catiónico destruido por la lixiviación de las bases y se reduce la acidez excesiva de los suelos (eleva el $\mathrm{pH}$ ) reduce la inmovilización del fósforo asimilable aplicado como fertilizante y favorece la actividad microbiana (Tahal Consulting, 1978). 
Cuadro 6. Costos de aplicación de fertilización mineral tradicional y costos de aplicación de abono orgánico gallinaza.

- Costos del insumo

$333,18 \mathrm{~kg}(7,33 \mathrm{qq})$ de $15-15-15 \times$ US $\$ 14,83=$ US $\$ 108,70$

$110,90 \mathrm{~kg}(2,44 \mathrm{qq})$ de urea $46 \%$ x US $\$ 15,50=$ US $\$ 37,82$

Total $=$ US\$146,52

- Costos de transporte, cargue y descargue

$$
444,09 \mathrm{~kg}(9,77 \mathrm{qq}) \quad \mathrm{x} \text { US } \$ 1,34=\mathrm{US} \$ 13,09
$$

- Costos de acarreo a la plantación (Combustible + panguero)

$$
444,09 \mathrm{~kg}(9,77 \mathrm{qq}) \quad \mathrm{x} \text { US\$ } 0,36 \quad=\text { US\$ } 3,51
$$

- Costos de aplicación (mano de obra)

$444,09 \mathrm{~kg}(9,77 \mathrm{qq})$ entre 2

$=4,8 \mathrm{~d} / \mathrm{h} \times \mathrm{US} \$ 2,69$

$=\mathrm{US} \$ 12,91$

\section{- Total costos de aplicación del fertilizante químico}

= US $\$ 176.03 /$ ha/año.

- Costos de aplicación de 1 qq de gallinaza
1 saco
$=$ US\$ 0,20
Transporte
$=$ US\$ 1,34
Acarreo a la plantación
$=$ US\$ 0,36
Costos de aplicación
$=$ US\$ 0,27

Total de costos de aplicación de 1 qq de gallinaza
US\$ 176,03 entre 2,17
US\$ 81,11 entre 4 aplicaciones
$=$ US $\$ 81,11 \mathrm{qq} / \mathrm{ha} / \mathrm{año}$.
$=921,16 \mathrm{~kg}(20,27 \mathrm{qq})$ por aplicación
$921,16 \mathrm{~kg}(20,27 \mathrm{qq}) \times 454 \mathrm{~g} \times 100 \mathrm{lb}$
$=920,258 \mathrm{~g} / \mathrm{ha} /$ aplicación
920,258 g. entre 1,111 plantas
$=0,8283 \mathrm{~kg} /$ plt.$/$ aplicación

$=\mathrm{US} \$ 2,17$

plt. : planta

$\mathrm{d} / \mathrm{h}:$ días hombre

Valor del dólar (U.S.A. \$) en córdobas al 15 de mayo de 1994.

Cuadro 7. Ecuaciones de regresión representando los costos de la fertilización mineral comparado con la aplicación de gallinaza.

\begin{tabular}{lccccc}
\hline Variables & $\begin{array}{c}\text { Ecuación lineal } \\
\text { de regresión }\end{array}$ & $\begin{array}{c}\text { Sust.por valor } \\
\text { obtenido con } \\
\text { fert.mineral }(\mathbf{x})\end{array}$ & $\begin{array}{c}\text { Costos de acuer- } \\
\text { acuerdo } \\
\text { a la f. m. }\end{array}$ & $\begin{array}{c}\text { Costos de apli- } \\
\text { aplicación } \\
\text { del f.org. }\end{array}$ & $\begin{array}{c}\text { Porcentaje } \\
\text { (mayor la f.m.) }\end{array}$ \\
\hline \% fruct. & $\mathrm{y}=1.806+2.908 \mathrm{x}$ & 0,833 & $828,3 \mathrm{~g}$ & $379,1 \mathrm{~g}$ & 118 \\
no. frutos & $\mathrm{y}=0.214+2.357 \mathrm{x}$ & 0,637 & $828,3 \mathrm{~g}$ & $287,8 \mathrm{~g}$ & 187 \\
incr.en diámetro & $\mathrm{y}=21.705+1.891 \mathrm{x}$ & 1,462 & $828,3 \mathrm{~g}$ & $663,7 \mathrm{~g}$ & 24 \\
\hline
\end{tabular}

$\%$ fruct. : porcentaje de fructificación

Incr. : incremento

Sust. : sustituyendo

obt. : obtenido

fert. : fertilizante

f.m. : fertilizante mineral

f. org. fertilizante orgánico 
El material utilizado para este ensayo (gallinaza), presentó un contenido de $60 \%$ de calcio (Cuadro 2) .Esto se considera un factor de importancia en el eficiente resultado obtenido en la fructificación.

En el Cuadro 8, se muestran los resultados del análisis realizado en los suelos del ensayo después de finalizado el trabajo de campo.

Los resultados presentados muestran diferencias en cuanto al pH del suelo, estos suelos están clasificados de fertilidad medio a bajo en cuanto a su acidez. Se puede observar que el tratamiento químico (T4) provoca acidificación al suelo en comparación al testigo absoluto (T5), no así el tratamiento con 1,362 g de gallinaza (T3) que elevó los valores.

En cuanto al contenido de materia orgánica, los resultados demuestran que existe un mayor porcentaje en suelos donde se aplicó 1,362 g de gallinaza. El tratamiento con fertilizante mineral presentó similar comportamiento que el testigo absoluto.

El fósforo no demostró un comportamiento muy claro, de manera que el tratamiento tres $(1,362 \mathrm{~g}$ de gallinaza) resultó superior a los tratamientos cuatro (fertilizante químico) y cinco (testigo absoluto) en el caso del productor tres e inferior en el caso del productor uno y dos.

En cuanto a las bases intercambiables, el calcio aumentó en las parcelas de todos los productores con la aplicación de gallinaza. Se considera normal, tomando en cuenta que la gallinaza posee un $60 \%$ de calcio lo que probablemente elevó también el valor del pH.
En cuanto al potasio y al magnesio se puede constatar que en dos casos hubo un aumento en su contenido con la aplicación de gallinaza, mientras que en la parcela del productor tres se registró un menor contenido de magnesio con la aplicación del tratamiento.

Con respecto al contenido de aluminio se registró en la parcela del productor 1 y 2 , niveles de toxicidad (mayor de $3 \mathrm{mg} / 100 \mathrm{~g}$ ) de 6,0 y 6,4 mg/100g bajo los tratamientos con fertilizante químico y testigo absoluto. El rango de los valores de aluminio con aplicación de gallinaza fue de 0 a 1,7 mg/100g. Existe la posibilidad que con el aumento del $\mathrm{pH}$ se redujo significativamente el nivel de este elemento.

Para poder llegar a interpretaciones más confiables se deberían realizar los análisis con suficientes repeticiones con el fin de evaluarlos estadísticamente, sin embargo, por limitaciones económicas esto no fue posible.

Respecto a las observaciones comparativas de forma visual al momento de obtener las muestras en las parcelas que recibieron el tratamiento de 1,362 $\mathrm{g}$ de gallinaza, se constató mayor actividad de la fauna del suelo. La estructura del suelo resultó más suelto con presencia de galerías, color oscuro y en algunas muestras se encontraron lombrices de tierra (Lombricus terrestri Lawson y colaboradores). No se observó una capa de acumulación del abono orgánico lo que confirma una rápida descomposición del mismo, únicamente se observaron restos de cáscaras de huevo en la superficie del suelo. En la parcela con aplicación de fertilizante mineral y testigo absoluto se observó menor actividad de la fauna edáfica.

Cuadro 8. Características químicas de los suelos finalizado el experimento.

\begin{tabular}{|c|c|c|c|c|c|c|c|c|c|}
\hline \multirow[t]{2}{*}{ Prod } & \multirow[t]{2}{*}{ Trat } & \multirow{2}{*}{$\begin{array}{c}\text { pH } \\
\text { H2O }\end{array}$} & \multicolumn{2}{|c|}{$\%$} & \multirow{2}{*}{$\begin{array}{c}\text { ppm } \\
\mathbf{P}\end{array}$} & \multicolumn{3}{|c|}{ meq/100 g suelo } & \multirow{2}{*}{$\begin{array}{c}\mathrm{mg} / \mathbf{1 0 0} \mathrm{g} \text { suelo } \\
\mathrm{Al}\end{array}$} \\
\hline & & & MO & $\mathbf{N}$ & & $\mathbf{K}$ & $\mathrm{Ca}$ & Mg & \\
\hline \multirow{3}{*}{1} & $\mathrm{~T} 3$ & 4,6 & 3,0 & 0,15 & 0,5 & 0,40 & 9,0 & 3,8 & 1,7 \\
\hline & $\mathrm{T} 4$ & 4,3 & 2,9 & 0,14 & 1,6 & 0,28 & 7,0 & 2,4 & 6,0 \\
\hline & $\mathrm{T} 5$ & 4,4 & 2,8 & 0,14 & 1,1 & 0,24 & 6,0 & 2,5 & 6,4 \\
\hline \multirow{3}{*}{2} & $\mathrm{~T} 3$ & 6,4 & 2,7 & 0,13 & 0,5 & 0,31 & 15,5 & 5,0 & 0,0 \\
\hline & $\mathrm{T} 4$ & 4,9 & 1,8 & 0,09 & 1,1 & 0,29 & 10,0 & 3,5 & 0,5 \\
\hline & T5 & 5,3 & 1,8 & 0,09 & 1,6 & 0,31 & 8,0 & 3,0 & 6,0 \\
\hline \multirow{3}{*}{3} & $\mathrm{~T} 3$ & 5,3 & 3,1 & 0,15 & 2,8 & 0,37 & 10,0 & 3,6 & 0,3 \\
\hline & $\mathrm{T} 4$ & 4,7 & 2,8 & 0,14 & 1,0 & 0,15 & 9,0 & 4,8 & 0,6 \\
\hline & T5 & 4,8 & 2,9 & 0,15 & 1,1 & 0,33 & 9,0 & 3,9 & 0,0 \\
\hline
\end{tabular}

PROD: Productor

TRAT: Tratamiento

Fuente: Analisis de suelo realizado en el Laboratorio de Suelos y Aguas de la Universidad Nacional Agraria (1994). 


\section{Análisis de regresión y correlación de las variables de suelo}

El análisis de regresión realizado entre las variables de suelo $\mathrm{Ca}, \mathrm{Mg}$ y Al se encontró que existe alta correlación entre el contenido de aluminio y la cantidad de calcio en el suelo. Se encontró una ecuación y=10,830535 $0,64999 x$, un coeficiente de correlación de $r=0,692$ (Figura 9), el coeficiente de determinación $\mathrm{r} 2=0,479$ indica que un $47,9 \%$ de la variación del calcio está determinado por el contenido de aluminio en el suelo. Existe una correlación altamente significativa entre el contenido de aluminio y el magnesio representado en la ecuación $\mathrm{y}=4,236611-0,26184 \mathrm{x}, \mathrm{r}=0,826, \mathrm{r} 2=0,682$. El $68 \%$ de la variación en el contenido de magnesio depende del contenido del aluminio en la solución del suelo. A mayor contenido de aluminio se reduce el contenido de calcio y magnesio en la solución del suelo.

Muestra la correlación que existe respecto a los contenidos de magnesio y calcio en el suelo, el comportamiento de la recta lineal de regresión mostró una ecuación $\mathrm{y}=0,856548+2,3320 \mathrm{x}$, el coeficiente encontrado mostró una correlación significativa de r= 0,787 un r $2=0,619$, indica que el $61,9 \%$ de la variación del contenido de magnesio en el suelo está determinado por el contenido de calcio en la solución del suelo.

\section{CONCLUSIONES}

Los resultados presentados y discutidos permiten concluir que:

1. La aplicación de abono orgánico (gallinaza) contribuyó al incremento del diámetro del tallo (aunque el análisis de varianza no detectó diferencias significativas). La prueba de Duncan a nivel de $5 \%$ de probabilidad muestra diferencias estadísticas significativas entre los tratamientos.

2. El tratamiento que mostró el mejor comportamiento en relación a la altura de planta fue el de $908 \mathrm{~g}$ de gallinaza, sin embargo, ésta variable se considera menos confiable, debido a que es más afectada por factores tanto genéticos, fisiológicos como antropogénicos.

3. En relación al porcentaje de floración se obtuvo un mayor efecto del tratamiento con 1,362 $\mathrm{g}$ de gallinaza, en comparación a los demás tratamientos, los tratamientos con $454 \mathrm{~g}$ de gallinaza y fertilizante químico (100 g de completo 15-15-15) presentaron similar comportamiento, estadísticamente no hubo diferencias.
4. La aplicación de $1,362 \mathrm{~g}$ de gallinaza resultó estadísticamente superior con respecto al resto de los tratamientos en lo que se refiere al análisis de porcentaje de fructificación después de un año de aplicación de los tratamientos estudiados.

5. Respecto al número de frutos prendidos se encontraron diferencias significativas entre el tratamiento con 1,362 $\mathrm{g}$ de gallinaza de aplicación con respecto al resto, mientras que los niveles $454 \mathrm{~g}, 908 \mathrm{~g}$ de gallinaza y fertilización química resultaron superiores con respecto al testigo absoluto.

6. El análisis económico mostró que la fertilización con abono orgánico gallinaza resultó más económica en comparación con la fertilización mineral tanto en el porcentaje de fructificación, número de frutos como en el incremento de diámetro de tallo. Según los cálculos de costos de fertilización se encontró que con los mismos costos de aplicación de fertilizante químico, es posible aplicar $0,82 \mathrm{~kg}$ $(1,8 \mathrm{lbs})$ de gallinaza por planta de cacao, para un total de $3,27 \mathrm{~kg}(7,2 \mathrm{lbs})$ al año y este material el productor lo puede adquirir en su propio hogar, si logra establecer un gallinero de 46 gallinas y recolectando el estiércol.

7. Según el análisis de suelo realizado se detectaron tendencias de aumento de la materia orgánica, del calcio y del $\mathrm{pH}$ en los suelos que recibieron el tratamiento con 1,362 g de gallinaza, lo que podría haber contribuido a la respuesta positiva en la fructificación de los árboles. Es importante señalar que un estudio de los cambios de los elementos edáficos requiere mayor tiempo y mayor cantidad.

\section{RECOMENDACIONES}

1. Se recomienda el uso de abonos orgánicos por las ventajas que éstos presentan, ya que es una alternativa para mejorar las condiciones edáficas y de esta manera aumentar la productividad en los cultivos y reducir la dependencia de fertilizantes químicos.

2. Con base al estudio realizado se recomienda sustituir la fertilización química con la aplicación de $0.82 \mathrm{~kg}$ (1.8 lbs) de gallinaza, considerando los costos de transporte. En caso de que este costo se reduce, deberán aplicarse $1.36 \mathrm{~kg}$ (tres libras) de gallinaza por árbol 3-4 veces al año.

3. Organizar los productores de la zona para transportar el material y utilizarlo en conjunto. 
4. Los organismos de transferencia de tecnología agrícola, deberán brindar asistencia técnica con orientación a un buen uso de los materiales orgánicos.

5. Continuar estudios para determinar el efecto del fertilizante orgánico en cacao en estado adulto y ampliar las investigaciones hacia otras materias orgánicas factibles para usarse en el cultivo de cacao.

6. Ampliar estudios en plantaciones cacaoteras de otras zonas del atlántico de Nicaragua, con materiales orgánicos, con el fin de evaluar el comportamiento de éstos en las diferentes zonas del país.

\section{LITERATURA CITADA}

AGRICULTURA DE LAS AMERICAS. 1965. La gallinaza es valiosa como fertilizante. 1014 Wyandotte Street,Kansas City, Missouri. E.U.A. University of Florida Texas A. \& M. University. Vol 1, pag. 20, Año 14, No. 1

ARZOLA, N.; FUNDORA, O.; MACHADO, J. 1986. Suelo, Planta y Abonado. Editorial Pueblo y Educación. Primera reimpresión. Habana, Cuba. 461 p.

BLANCO, M. 1984. Cultivos Industriales. Universidad Nacional Autónoma de Nicaragua. Facultad de Ciencias Agropecuarias. Editorial Pueblo y Educación de Cuba para el CNES, Managua, Nic. 211 p.

COMPAÑÍA NACIONALDECHOCOLATES, 1988. Manual para el cultivo de cacao. EDINALCO. Tercera edición. Colombia. 140 p.
HOLDRIDGE, L.R. 1967. Life Zone Ecology (2 Aufl) Trop. Res. Center, San José, Costa Rica. 206 p.

ICCO, 1,993. Boletín Trimestral de Estadísticas del Cacao. Organización Internacional del cacao. 22 Barners Street London wip 3db United Kingdom.

MALESPIN, M. 1982. El cacao. Ministerio de Desarrollo Agropecuario y Reforma Agraria. IICA. Fondo Simón Bolívar 61p.

TAHAL CONSULTING. 1978. Potencial de desarrollo agropecuario y rehabilitación de tierras en la costa Atlántica-Nicaragua. Dirección de Planificación Nacional. Informe Principal. Vol 1, Pág. 17, 187 p.

THIENHAUS, S. 1986. Informe anual, Comparación de diferentes fuentes de fertilizantes en cultivo de cacao. Centro Experimental El Recreo, Km 279 carretera a Rama. $12 \mathrm{p}$.

THIENHAUS, S. 1992. Diagnóstico nacional del cultivo de cacao en Nicaragua. Primera edición, 70 p.

U.N.A. 1993. Laboratorio de suelos y aguas de la Universidad Nacional Agraria. p. 7 y 8.

U.N.A. 1994. Laboratorio de suelos y aguas de la Universidad Nacional Agraria. p. 34.

VOITL/ GUGGENBERGER/ WILLI, 1980. Das Grosse Buch vom Biologischen Land- und Gartenbau. 367 p.

YÁODIN, B.A. 1982. Agroquímica II. Editorial Moscú. Traducido alEspañol, editorial Mir, 1982.pag. 120.464p. 